\title{
Effects of Pelvic Compression Belt on Three-dimensional Motions of Pelvic and Trunk during One Leg Standing
}

\author{
Tae-ho Kim, Ph.D; Woo-min Je, MSc; Byoung-ha Hwang, Ph.D \\ Department of Physical Therapy, College of Rehabilitation Science, Daegu University, Daegu, South Korea
}

Background Low back pain caused lumbopelvic instability and the passive and active stabilizing system and neural control subsystem control lumbopelvic stability.

Purpose The aim of this study was to identify three-dimensional motions of pelvic and trunk during one leg standing applying the pelvic compression belt.

Study design One group pre- and post-test design.

Methods Twenty three healthy participants volunteered for this study. For the pre-test, their pelvic and trunk motions were measured during one leg standing in stance of the dominant side using equipment without application of pelvic compression belt. The participants wore pelvic compression belt for 20 minutes on a daily basis for a week in standing position to get used to application of pelvic compression belt. After one week training, the participants were measured during one leg standing in stance of the dominant side using equipment with application of pelvic compression belt for the post-test. The three-dimensional motion analysis system was used to measure the motion of anterior-posterior tilt, medial-lateral tilt, and rotation of the pelvis, as well as anterior-posterior flexion, lateral flexion, and rotation of the trunk during one leg standing.

Results The motion of anterior-posterior tilt, medial-lateral tilt, and rotation of the pelvis were significantly decreased in the post-test than pre-test during one leg standing $(p<0.05)$. Also the motion of anterior-posterior flexion, lateral flexion, and rotation of the trunk were significantly decreased in the post-test than pre-test during one leg standing $(p<0.05)$. Effect sizes for pelvic anterior-posterior tilt, anterior-posterior flexion, lateral flexion, and rotation of the trunk were medium between pre-test and post-test during one leg standing $(d=055-0.75)$.

Conclusions This study found the motions of the pelvis and trunk were decreased by the passive support of the pelvic compression belt during one leg standing. This finding suggests that the pelvic compression belt will help to improve the stability of lumbopelvis in back pain patients with lumbopelvic instability.

Key words Lumbopelvic stability; One leg standing; Pelvic compression belt, Pelvic motion.

\section{INTRODUCTION}

One leg standing is a necessary sequence for dynamic transitions of body weight during walking and is required for a number of daily activities. And it is as a method of test used to assess the ability and movement quality of the sacroiliac joint for maintenance of lumbopelvic stability
JMST

2019; 3(1): 22-25

Published Online Jun 30, 2019

pISSN 2635-8573 eISSN 2635-8581

\section{Article History}

Received 15 April 2019

Revised 15 April 2019 (1st)

Revised 05 May 2019

(2nd)

Accepted 10 May 2019

\section{CONTACT}

hohoho90@naver.com

Woo-min Je,

Department of Physical

Therapy, College of

Rehabilitation Science,

Daegu University,

Daegu , South Korea

This is an Open-Access article distributed under the terms of the Creative Commons Attribution Non-Commercial License (http://creativecommons. org/licenses/by-nc/4.0) which permits unrestricted non-copermits unrestricted non-coand reprom ais reproduction in any medium, provided the original work is properly cited. during the transmission of load between the lower extremities and the spine. ${ }^{1,2}$ According to previous studies, the alteration of the muscular activation pattern easily not only affected functional and daily activities but caused lumbopelvic instability, and lumbopelvic instability caused pelvic anterior rotation due to asymmetrical hip flexion during load transfer such as one leg standing. ${ }^{3,4}$ 
The passive support for lumbopelvic region could be optimized by therapeutic exercises with a pelvic compression belt (PCB). A previous study reported that PCB influenced sacroiliac joint laxity and lumbosacral angle as well as lumbopelvic muscle strength, muscle activity and pain. $^{5}$ It demonstrated that $\mathrm{PCB}$ compressed the articular surface of the sacroiliac joint and increased stability on sacroiliac joint and lumbopelvic region. Use of the PCB also enabled performance of active straight leg raising test with few difficulties in women with pelvic girdle pain. ${ }^{6}$ Therefore, it is often recommended that patients suffering from sacroiliac joint pain wear the PCB while walking and standing.

Many researchers have investigated the effect of PCB on diverse variables including the active straight leg raising test, muscle activity associated with lumbopelvic region and hip joint, muscle activation patterns, biomechanical influence of muscles and ligament forces, form closure, force closure and neuromotor control., ${ }^{3,5}$ There have been some studies to investigate the correlation of one leg standing with motions of pelvis and trunk ${ }^{1,7}$ or factors influencing motions during one leg standing ${ }^{8}$, however no study applying PCB respectively to stabilize lumbopelvic region in association with motions during one leg standing has been reported. The aim of this study was to identify threedimensional motions of pelvic and trunk during one leg standing applying the pelvic compression belt.

\section{METHODS}

\section{Participants}

Twenty three healthy participants $(\mathrm{M}=15, F=8)$ read consent and volunteered for this study. The average age of participants was $25.00 \pm 2.39$ years, the height was 170.70 $\pm 8.45 \mathrm{~cm}$, the weight was $65.97 \pm 11.84 \mathrm{~kg}$, and dominant leg was 12 right side and 11 left side. Exclusion criteria included the history or presence of pain on back, pelvis, leg or foot, as well as of deformities, leg length discrepancy, acute trauma, surgery for the lower extremities or back, sacroiliac joint or balance impairment. This study was approved by the Institutional Review Board of Daegu University.

\section{Measurement of three-dimensional motion}

The three-dimensional motion analysis system (CMS 70P, Zebris Medizintechnik, Gmbh, Isny, Germany) was used to measure the motion of anterior-posterior tilt, medial-lateral tilt, and rotation of the pelvis, as well as anterior-posterior flexion, lateral flexion, and rotation of the trunk during OLS.
A set of triple markers for measurement of motions of the pelvis was placed at the midpoint between right and left ASIS using an elastic Velcro strip. Other triple markers were aligned vertically to the midline of the trunk to measure motions of the trunk. The measuring sensor was located at a distance of 1 meter from the participant. ${ }^{8}$ The sampling rate for this study was $20 \mathrm{~Hz}$ and used 6 markers, 6 angles, and 80 degrees of inclination. Windata 2.19.44 software was used for data analysis. The unit of motion was degree $\left({ }^{\circ}\right)$.

\section{Procedure}

For the pre-test, their pelvic and trunk motions were measured during one leg standing in stance of the dominant side using equipment without application of PCB. The PCB (JEONGLIB O\&P Inc., Seoul, Korea)(Figure 1) consists of the elastic band type and inelastic Velcro type. The participants wore PCB for 20 minutes on a daily basis for a week in standing position to get used to application of PCB. After one week training, the participants were measured during one leg standing in stance of the dominant side using equipment with application of $\mathrm{PCB}$ for the post-test. The PCB was positioned just below the ASIS. ${ }^{9}$ In the present study, the PCB was maximally tightened by an examiner without any discomfort to participants.

For the starting position of the one leg standing, the participant was asked to maintain the standing position comfortably, with both feet on the line for 5 seconds. When the examiner signaled, the subject lifted the non-dominant hip, which was flexed to 60 degrees in the sagittal plane, while the lower leg was hung with the ankle joint vertically in a relaxed position for 5 seconds (Figure 1). If the lifted foot touched the standing leg or the floor it was considered that balance was disturbed, and the one leg standing test was repeated. A resting period of 1 minute was provided between trials and the mean value of three trials was used for statistical analysis. All participants were asked to become

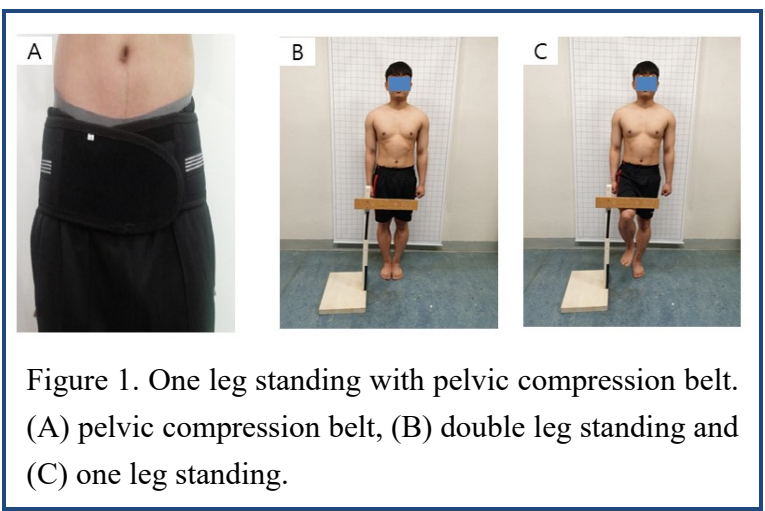


accustomed to standing with one leg before initiation of the one leg standing test.

\section{Statistical analysis}

The Shapiro-Wilk test was used to test for a normal distribution. The paired $t$-test was used to compare the differences in pelvic and trunk motions between pre-test and post-test. Cohen's d was calculated to measure the size of the mean difference between pre-test and post-test. The level of statistical significance was set at $p<0.05$. The Statistical Package for the Social Sciences (SPSS) version 23.0 (SPSS, Inc., Chicago, IL, USA) software was used for the statistical analysis.

\section{RESULTS}

The motion of anterior-posterior tilt $(-1.65 \pm 1.97)$, medial-lateral tilt $(-0.77 \pm 1.32)$, and rotation $(-0.70 \pm 1.53)$ of the pelvis were significantly decreased in the post-test than pre-test during one leg standing $(p<0.05)$ (Table 1) (Figure 2). Also the motion of anterior-posterior flexion ($2.36 \pm 2.12)$, lateral flexion $(-1.60 \pm 1.77)$, and rotation ($1.31 \pm 1.56)$ of the trunk were significantly decreased in the post-test than pre-test during one leg standing $(p<0.05)$ (Table 1) (Figure 2). Effect sizes for pelvic anteriorposterior tilt, anterior-posterior flexion, lateral flexion, and rotation of the trunk were medium between pre-test and post-test during one leg standing $(d=055-0.75)$ (Table 1$)$

\section{DISCUSSION}

The study aimed to identify three-dimensional motions of pelvic and trunk during one leg standing applying the pelvic compression belt. All motions of pelvis and trunk were significantly decreased after applying the pelvic compression belt during one leg standing. Arumugam et al reported that the PCB can provide stability to the lumbopelvic region and the application of PCB influenced sacroiliac joint laxity

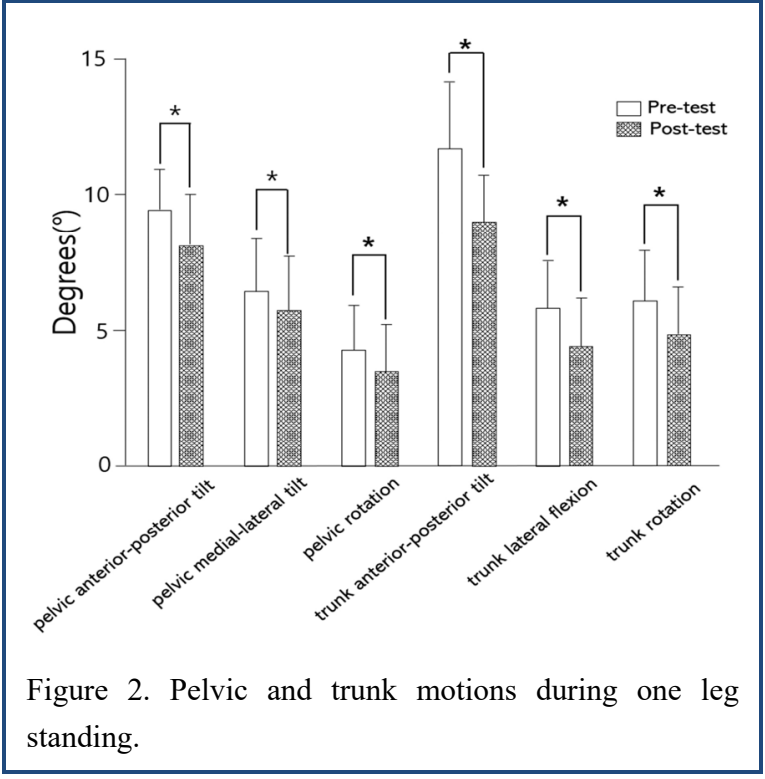

and mobility by compressing the articular surface of the sacroiliac joint. ${ }^{5}$ It was considered that the passive support of the PCB contributed to lumbopelvic stability and resulted in the decreases of all pelvic and trunk motions during one leg standing.

Previous studies have reported that lumbopelvic stability is controlled by three subsystems. Osseo-ligaments and capsules in the spinal column achieve the passive stabilizing system, while active stabilizing is achieved by spinal muscles and neural control subsystems. ${ }^{10,11}$ Dysfunction within any of these systems could result in an injury to a component of the system, compensation of the other systems, or long-term adaptations, all of which could represent causes of impaired lumbopelvic stability. ${ }^{10}$ Several researchers have shown that lumbopelvic stability is contributed by the longitudinal sling such as multifidus and long head of biceps femoris, posterior oblique sling such as latissimus dorsi and gluteus maximus and anterior oblique sling such as external oblique, internal oblique, and transversus abdominis. ${ }^{12-14}$ In an optimal stability of the

Table 1. Comparison for pelvic and trunk motions in pre-test and post-test

$(n=23)$

\begin{tabular}{lccccc}
\hline \multicolumn{1}{c}{ Motions $\left(^{\circ}\right)$} & Pre-test & Post-test & $t$ & $p$ & Effect size $d$ \\
\hline Pelvic anterior-posterior tilt & $9.76 \pm 1.24$ & $8.11 \pm 1.88$ & 4.01 & $0.00^{*}$ & 0.73 \\
Pelvic medial-lateral tilt & $6.79 \pm 2.19$ & $6.02 \pm 2.23$ & 2.80 & $0.01^{*}$ & 0.25 \\
Pelvic rotation & $4.59 \pm 1.35$ & $3.89 \pm 1.58$ & 2.18 & $0.04^{*}$ & 0.34 \\
Trunk anterior-posterior flexion & $11.49 \pm 3.37$ & $9.13 \pm 2.62$ & 5.33 & $0.00^{*}$ & 0.55 \\
Trunk lateral flexion & $6.10 \pm 1.50$ & $4.50 \pm 1.62$ & 4.35 & $0.00^{*}$ & 0.72 \\
Trunk rotation & $6.35 \pm 1.85$ & $5.04 \pm 1.52$ & 4.03 & $0.00^{*}$ & 0.55 \\
\hline
\end{tabular}


lumbopelvic motion, neural components which are various central strategies continually control and modulate the muscle recruitment pattern and timing as the active system to provide the stability. ${ }^{4,10}$ Although this study did not confirm muscle activity of trunk muscles during one leg standing applying the PCB, the PCB would have assisted passive stabilizing system and active stabilizing system in lumbopelvic region by elastic tension of the compression belt.

This study has some limitations. The participants for this study were healthy subjects without back pain and this study did not examined the muscle activities of trunk including external oblique, internal oblique, and transversus abdominis during one leg standing. Due to the passive support of the pelvic compression belt, the reduced motions of the pelvis and trunk ranged between 0.7 degree and 2.36 degree. Although the angles have decreased statistically significantly, it is important to consider how the values of the angle are clinically meaningful. In further study, it is necessary to confirm the effects of the pelvic compression belts on the back pain patients with muscle activities of trunk.

\section{CONCLUSIONS}

This study found the motions of the pelvis and trunk were decreased by the passive support of the pelvic compression belt during one leg standing. This finding suggests that the pelvic compression belt will help to improve the stability of lumbopelvis in back pain patients with lumbopelvic instability.

\section{Key Points}

Question Does the pelvic compression belt decrease motions of pelvic and trunk during one leg standing?

Findings All motions of pelvis and trunk were significantly decreased after applying the pelvic compression belt.

Meaning The pelvic compression belt reduce the motions of the pelvis and trunk and it will help to improve the lumbopelvic stability.

\section{Article information \\ Conflict of Interest Disclosures: None. \\ Funding/Support: None. \\ Acknowledgment: None.}

\section{REFERENCES}

1. Luomajoki H, Kool J, de Bruin ED, et al. Reliability of movement control tests in the lumbar spine. $B M C$ Musculoskelet Disord. 2007;8:90-100.

2. Tidstrand J, Horneij E. Inter-rater reliability of three standardized functional tests in patients with low back pain. BMC Musculoskelet Disord. 2009;10:58-66.

3. $\mathrm{Hu} \mathrm{H}$, Meijer OG, van Dieen JH, et al. Muscle activity during the active straight leg raise (ASLR), and the effects of a pelvic belt on the ASLR and on treadmill walking. J Biomech. 2010;43(3):532-539.

4. Hungerford B, Gilleard W, Hodges P. Evidence of altered lumbopelvic muscle recruitment in the presence of sacroiliac joint pain. Spine. 2003;28(14):1593-1600.

5. Arumugam A, Milosavljevic S, Woodley S, et al. Effects of external pelvic compression on form closure, force closure, and neuromotor control of the lumbopelvic spine-a systematic review. Man Ther. 2012;17(4):275284.

6. Mens JM, Damen L, Snijders CJ, Stam HJ. The mechanical effect of a pelvic belt in patients with pregnancy-related pelvic pain. Clin Biomech (Bristol, Avon). 2006;21(2):122-127.

7. Sahrmann SA. Diagnosis and treatment of movement impairment syndromes. St Louis, MO: Mosby Inc; 2002

8. Kim TH. Factors influencing pelvic and trunk motions during one-leg standing. Won-ju, Korea, University of Yonsei, Doctorial dissertation; 2010.

9. Damen L, Spoor CW, Snijders CJ, et al. Does a pelvic belt influence sacroiliac joint laxity? Clin Biomech (Bristol, Avon). 2002;17(7):495-498.

10. Panjabi MM. The stabilizing system of the spine. part I. function, dysfunction, adaptation, and enhancement. $J$ Spinal Disord. 1992;5(4):383-389.

11. Panjabi MM. Clinical spinal instability and low back pain. J Electromyogr Kinesiol. 2003;13(4):371-379.

12. Pool-Goudzwaard AL, Vleeming A, Stoeckart R, et al. Insufficient lumbopelvic stability: a clinical, anatomical and biomechanical approach to 'a-specific'low back pain. Man Ther. 1998;3(1):12-20.

13. Snijders CJ, Ribbers MT, de Bakker HV, et al. EMG recordings of abdominal and back muscles in various standing postures: validation of a biomechanical model on sacroiliac joint stability. J Electromyogr Kinesiol. 1998;8(4):205-214.

14. Vleeming A, Pool-Goudzwaard AL, Stoeckart R, et al. The posterior layer of the thoracolumbar fascial its function in load transfer from spine to legs. Spine. 1995;20(7):753-758. 